\title{
Correction to: Trials with patient-reported outcomes registered on the Australian New Zealand Clinical Trials Registry (ANZCTR)
}

\author{
Rebecca Mercieca-Bebber ${ }^{1,2,3}$ - Douglas Williams ${ }^{2} \cdot$ Margaret-Ann Tait $^{2} \cdot$ Jessica Roydhouse ${ }^{4} \cdot$ Lucy Busija $^{5}$. \\ Chindhu Shunmuga Sundaram ${ }^{2} \cdot$ Michelle Wilson $^{6,7} \cdot$ Ailsa Langford $^{3}$. Claudia Rutherford ${ }^{2} \cdot$ Natasha Roberts $^{8,9}$. \\ Madeleine King ${ }^{1,2}$. Elisabeth Vodicka ${ }^{10} \cdot$ Beth Devine ${ }^{10,11,12}$ on behalf of the International Society for Quality of Life \\ Research (ISOQOL)
}

Published online: 16 July 2018

(c) Springer Nature Switzerland AG 2018

\section{Correction to: Quality of Life Research https://doi.org/10.1007/s11136-018-1921-5}

In the original publication of the article, the sentence "The ANZCTR is the fifth largest trial registry internationally, with $\mathbf{2 1 , 3 3 0}$ registered trials as at January 2018 [5]" in the
Introduction section was published incorrectly. It should say: "The ANZCTR is the fifth largest trial registry internationally, with $\mathbf{1 4 , 9 8 8}$ registered trials as at January 2018 [5]".

The original article can be found online at https://doi.org/10.1007/ s11136-018-1921-5.

Rebecca Mercieca-Bebber

Rebecca.Mercieca@sydney.edu.au

Douglas Williams

Douglas.williams@sydney.edu.au

Margaret-Ann Tait

margaret-ann.tait@sydney.edu.au

Jessica Roydhouse

jessica_roydhouse@brown.edu

Lucy Busija

Lucy.Busija@acu.edu.au

Chindhu Shunmuga Sundaram

cshu3384@uni.sydney.edu.au

Michelle Wilson

MiWilson@adhb.govt.nz

Ailsa Langford

ailsa.langford@ctc.usyd.edu.au

Claudia Rutherford

Claudia.Rutherford@sydney.edu.au

Natasha Roberts

Natasha.roberts@healht.qld.gov.au

Madeleine King

Madeleine.King@sydney.edu.au

Elisabeth Vodicka

evodicka@uw.edu

Beth Devine

bdevine@uw.edu
1 Faculty of Medicine, Sydney Medical School, Central Clinical School, University of Sydney, Sydney, Australia

2 Faculty of Science, School of Psychology, University of Sydney, Sydney, Australia

3 NHMRC Clinical Trials Centre, University of Sydney, Sydney, NSW 2006, Australia

4 Department of Health Services, Policy \& Practice, Brown University School of Public Health, Providence, RI, USA

5 Institute for Health \& Ageing, Australian Catholic University, Melbourne, Australia

6 Auckland City Hospital, Auckland, New Zealand

7 University of Auckland, Auckland, New Zealand

8 Royal Brisbane and Women's Hospital (RBWH), Herston, Australia

9 Queensland University of Technology (QUT), Brisbane, Australia

10 Comparative Health Outcomes, Policy, and Economics (CHOICEc) Institute, University of Washington, Seattle, WA, USA

11 Department of Biomedical Informatics and Medical Education, University of Washington, Seattle, WA, USA

12 Department of Health Services, University of Washington, Seattle, WA, USA 